\title{
The expression of podoplanin is associated with poor outcome in cutaneous squamous cell carcinoma
}

Background: Cutaneous squamous cell carcinoma (CSCC) is the second most frequent cancer in humans and can be both locally invasive and metastatic at distant sites. While research efforts have been made to predict poor outcome of CSCC, there is a lack of knowledge regarding molecular markers. Podoplanin has been associated with poor outcome in several types of cancer including CSCC, but this is controversial and only a few studies have evaluated the prognostic implications of podoplanin in the development of this tumor.

Methods: We evaluated podoplanin expression in a series of 94 CSCCs, and searched for associations between podoplanin expression and histopathological characteristics and with events of poor clinical evolution of the disease.

Results: Podoplanin expression was observed in $48.9 \%$ of the cases and the expression was considered moderate to intense in 19 of the cases. Moderate/intense podoplanin was associated with infiltrative growth pattern, desmoplasia, lymphovascular invasion, higher risk of nodal progression (NP) and short disease-free survival, specifically with a short latency to NP.

Conclusions: This article provides evidence supporting the implication of podoplanin expression as a marker of bad prognosis of CSCC.

Keywords: cutaneous squamous cell carcinoma, D2-40, podoplanin, prognosis

Cañueto J, Cardeñoso-Álvarez E, Cosano-Quero A, Santos-Briz Á, Fernández-López E, Pérez-Losada J, Román-Curto C. The expression of podoplanin is associated with poor outcome in cutaneous squamous cell carcinoma.

J Cutan Pathol 2017; 44: 144-151. (C) 2016 John Wiley \& Sons A/S. Published by John Wiley \& Sons Ltd

\section{Javier Cañueto ${ }^{1,2}$, Ester Cardeñoso-Álvarez ${ }^{3}$, Adriana Cosano-Quero', Ángel Santos-Briz ${ }^{4}$, Emilia Fernández-López ${ }^{1,2}$, Jesús Pérez-Losada ${ }^{2,5, \dagger}$ and Concepción Román-Curto ${ }^{1,2, \dagger}$}

${ }^{1}$ Servicio de Dermatología, Hospital Universitario de Salamanca, Salamanca, Spain,

${ }^{2}$ Instituto de Investigación Biomédica de Salamanca (IBSAL), Hospital Universitario de Salamanca, Salamanca, Spain,

${ }^{3}$ Servicio de Dermatología, Hospital Virgen de la Concha, Zamora, Spain,

${ }^{4}$ Servicio de Patología, Hospital Universitario de Salamanca, Salamanca, Spain, and

${ }^{5}$ Instituto de Biología Molecular y Celular del Cáncer (IBMCC), Centro de investigación del

Cáncer (CIC), Universidad de

Salamanca/CSIC, Salamanca, Spain

†These authors contributed equally as senior authors.

Dr Javier Cañueto,

Department of Dermatology, University Hospital

of Salamanca, Paseo San Vicente, 58-182,

37007 Salamanca, Spain

Tel: +34 923291100 ext. 55446

Fax: +34 923294813

e-mail: jcanueto@yahoo.es

Accepted for publication November 1, 2016
Cutaneous squamous cell carcinoma (CSCC) represents the most common form of skin cancer after basal cell carcinoma and can be both locally invasive and metastatic at distant sites. ${ }^{1,2}$ Around 700,000 CSCCs new cases are diagnosed annually in the United States, comprising a proportion of
$20 \%$ of all non-melanoma skin cancer (NMSC). ${ }^{3}$ While most CSCC cases have an excellent prognosis, there is a subset with a high risk of developing local recurrence (LR) and metastases. The risk of nodal metastases ranges from 3.7 to $5.2 \%$, and disease-specific death ranges 


\section{D2-40 in cutaneous squamous cell carcinoma prognosis}

from 1.5 to $2.1 \%$ in CSCG patients. ${ }^{4}$ Given the high frequency of CSCC and its increasing incidence, the clinical implications of CSCC are of paramount importance and its associated costs are high. ${ }^{5}$ Several clinical and histopathological risk factors have been used to predict bad clinical evolution in patients diagnosed with CSCC, ${ }^{6-8}$ but there is an evident lack of knowledge concerning molecular and protein markers that could be used to predict outcome of this disease.

Podoplanin is essential in lymphatic vessel development ${ }^{9}$ and also participates in tumor invasion and progression. ${ }^{10}$ It was originally identified as a cell-surface antigen expressed in mouse epidermal keratinocytes during skin remodeling and carcinogenesis. ${ }^{11}$ Podoplanin expression was also linked to epidermal to mesenchymal transition (EMT), cell migration ${ }^{12}$ and to an alternative pathway for tumor cell invasion in the absence of EMT. ${ }^{13}$ Podoplanin is also involved in the actin remodeling of the cytoskeleton of tumor cells and may promote tumor cell invasion by increasing cell motility. ${ }^{13}$

Podoplanin expression has been associated with poor outcome in some types of cancer. Podoplanin is upregulated in several human cancers such as squamous cell carcinoma (SCC) of the oral cavity, ${ }^{14-16}$ skin adnexal carcinomas, ${ }^{17}$ esophageal SCC ${ }^{18}$ and SCC of the penis. ${ }^{19}$ Some papers have showed that podoplanin expression is associated with a higher risk of nodal metastasis in CSCC, ${ }^{20,21}$ but there is still debate regarding its real relevance as a prognostic marker. This study provides further evidence regarding the prognostic implications of podoplanin expression in primary CSCCs, and highlights its relevance in predicting disease-free survival.

\section{Patients, material and methods}

Clinical, epidemiological and histopathological features of CSCCS

We evaluated a series of 94 CSCCs. We selected some tumors in a prospective way, and other tumors were retrospectively collected in order to obtain a higher proportion of CSCC with poor outcome and high risk features. Patients were included following the Helsinki Declaration and the requirements set out by the Local Bioethical Committee of the University Hospital of Salamanca. We considered different tumor and epidemiological variables of CSCCs, clinical-epidemiological and histopathological features and their distribution along the cohort are described on Table S1, Supporting Information. Specifically, we considered the following values:

\section{Clinical and epidemiological variables}

(a) Age and sex of patients; (b) Previous history of dermatological diseases, including actinic keratoses, NMSC and CSCC; (c) Tumor location was categorized as: (i) head and neck high-risk areas (ear, lower lip, temple, nose, eyelid and preauricular region); (ii) head and neck low-risk areas (rest of head and neck locations) and (iii) trunk and extremities; (d) Presence of immunosuppression; (e) History of chronic sun exposure and (f) American Joint Committee on Cancer (AJCC) stage at diagnosis and at the end of the follow-up period.

\section{Histopathological variables}

(a) Tumor size and thickness (in millimeters), both measured using the OV100 software (Olympus $^{\mathrm{TM}}$, Tokyo, Japan); (b) grade of differentiation, which was classified as good, moderate or poor, according to previous reports ${ }^{22}$; (c) growth pattern was classified as expansive, infiltrative or mixed. Infiltrative growth pattern was considered when the tumor exhibited small nests, rows of cells and/or isolated tumor cells in the periphery of the tumor, an expansive pattern was assigned when the tumor exhibited a compact growth, with no-disaggregated cells in the front of invasion and mixed growth pattern was assigned when the tumor displayed an expansive growth pattern and tended to infiltrate in any part of the front of invasion; (d) perineural invasion; (e) lymphovascular invasion; (f) desmoplasia was defined by the thickening of collagen bundles around and inside the tumor involving at least $30 \%$ of the stroma ${ }^{6} ;(\mathrm{g})$ solar elastosis and $(\mathrm{h})$ actinic keratosis associated with tumor (in flanking epithelium).

Time to events of poor clinical evolution during follow up (in months)

(a) LR; (b) NP; (c) distant progression, which was considered when the tumor developed metastases in solid organs during the follow-up period; (d) death from CSCC and (e) any of the aforementioned events. ${ }^{23}$

\section{Tissue microarray and immunohistochemistry}

Tissue samples, formalin-fixed paraffinembedded, were used to prepare tissue microarrays using a tissue arrayer device (Beecher 
Instruments, Sun Prairie, WI, USA). Three 1-mm-diameter cylinders from each tumor were included to ensure reproducibility and homogeneous staining of the slides. Three different areas of each tumor were selected to reproduce the heterogeneity of the samples.

Immunohistochemical studies were performed using the AutostainerLink 48 (Dako, Santa Clara, CA, USA). Antibody anti-human against podoplanin, clone D2-40 (Dako, Santa Clara, CA, USA) of mouse origin, was used (prediluted). The detection system used the kit EnVision/HRP Flex Plus (Dako ${ }^{\circledR}$, Santa Clara, CA, USA) and positive controls were included in each slide.

A semiquantitative analysis of podoplanin expression level was performed by three independent observers (JC, CR-G and EC-A). The intensity of podoplanin expression and the percentage of positive cells in each tumor were calculated as carried out by other groups. ${ }^{20,21}$ Podoplanin intensity of expression was calculated as 0 : null; 1: weak; 2: moderate and 3: intense; and the percentage of positive cells was considered as 0 : null ( $<25 \%$ of stained cells); 1 : weak $(25-50 \%)$; 2: moderate $(50-75 \%)$ and 3 : intense $(>75 \%)$. A mean with podoplanin intensity of expression and podoplanin percentage of stained cells were calculated to determine the amount of podoplanin expression in each tumor, as previously described. ${ }^{21}$ Podoplanin expression was later transformed into a dichotomous variable with category 0 (absent and weak podoplanin expression: $-/+$ ) and category 1 (moderate and intense podoplanin expression: $++/+++)$. We only considered podoplanin expression in epithelial tumor cells in order to classify CSCG. ${ }^{20,21}$

Lymphovascular invasion was evaluated and considered positive when tumor cells (either isolated or organized in clusters) were surrounded by podoplanin-positive lymphatic vessels.

\section{Statistical analysis}

Statistical analysis was performed using the IBM SPSS v.21 software. Categorical variables were compared using the Chi-square or the Fisher exact test when appropriate. The Kolmogorov-Smirnov test showed that continuous variables did not follow a normal distribution. When two independent samples were analyzed, the Mann-Whitney $U$ test was used. To evaluate which variables predicted events of poor clinical evolution, we developed logistic regression models and used the Wald test. We considered $\mathrm{p}$ values $<0.05$ as significant, and confidence intervals at $95 \%$.

\section{Results}

\section{Podoplanin expression in CSCC}

To determine the utility of podoplanin expression as a marker of prognosis in CSCC, we evaluated podoplanin expression by immunohistochemistry in a cohort of 94 CSCCs (Fig. 1 and Table 1). A total of 48 tumors $(51.1 \%)$ displayed no expression, 27 tumors $(28.8 \%)$ showed weak expression, 15 tumors (16\%) showed moderate expression and 4 tumors $(4.3 \%)$ displayed intense expression of podoplanin (Table 1). Podoplanin expression was located mainly in lymphatic vessels, but in some cases also in tumor cells in which podoplanin exhibited a cytoplasmic staining, sometimes with cell membrane enhancement. Podoplanin also tended to be expressed along the front of invasion of the tumors, especially in those cases with an infiltrative growth pattern (Fig. 1).

Podoplanin expression is associated with histopathological tumor traits and with events of poor outcome in CSCC

Following on, we studied the existence of associations between podoplanin expression and histopathological tumor traits. Moderate/intense podoplanin expression was associated with the presence of desmoplasia $(p=0.031)$, with an infiltrative growth pattern $(p=0.013)$ and with the presence of lymphovascular invasion $(p=0.011)$. Moderate/intense podoplanin expression was also found more frequently in those tumors that exhibited ulceration $(p=0.002)$ and a poor grade of differentiation with a statistical trend $(p=0.057)$ although, expression was not associated with perineural invasion (Fig. 2 and Table 1).

Moderate/intense podoplanin expression was not associated with AJCC stage at diagnosis but it correlated with AJCC stage at the end of follow up. Thus, podoplanin expression was more common in tumors at the higher stages $(p=0.032)$ and with progression of the AJCC stage $(p=0.022)$. More interestingly, moderate/intense podoplanin expression in the primary tumor correlated with higher risk of nodal metastasis during follow up $(p=0.022)$ (Table 1), which was particularly evident for tumors at stage $2(p=0.01)$ (Fig. 2C). 


\section{D2-40 in cutaneous squamous cell carcinoma prognosis}
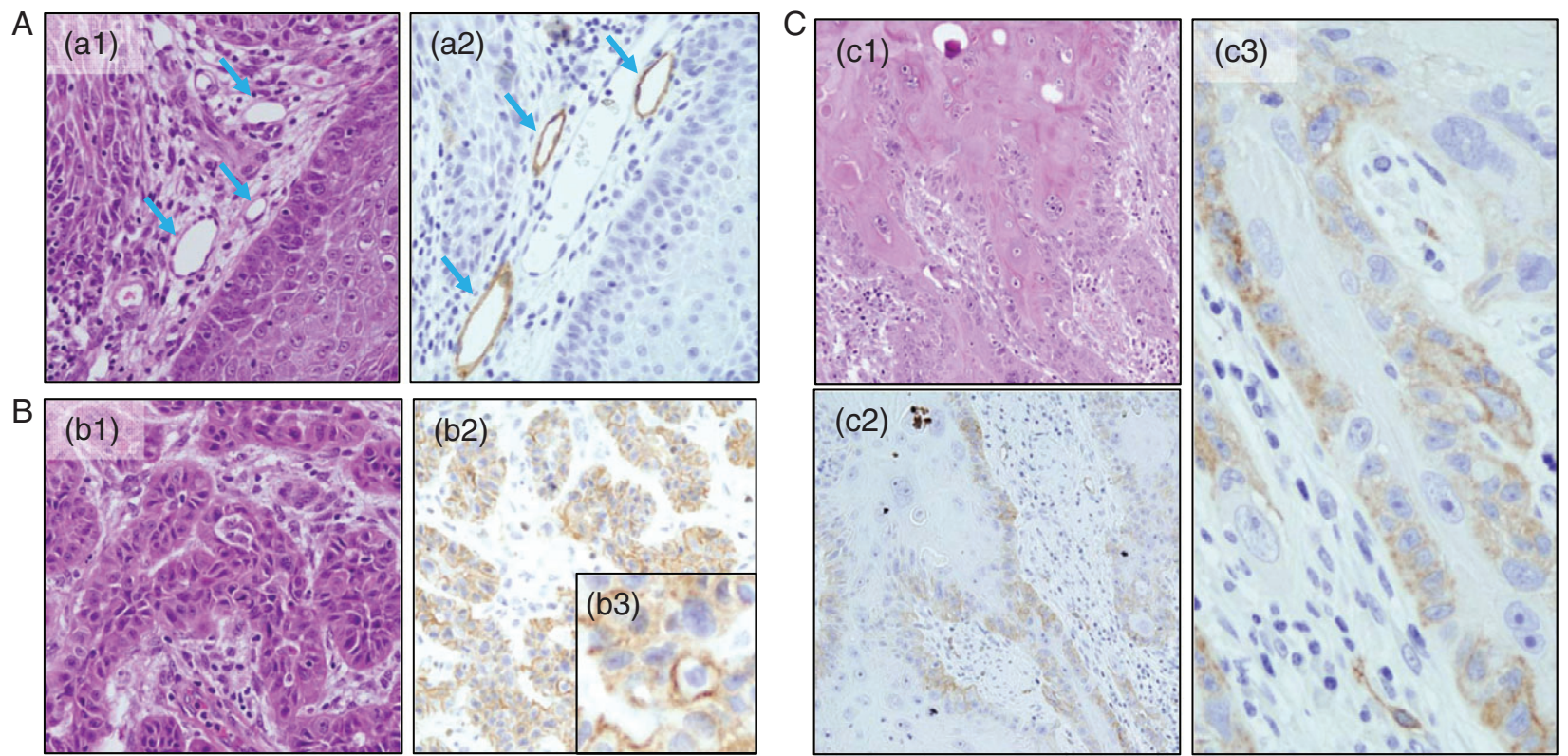

Fig. 1. Evaluation of podoplanin expression by immunohistochemistry in cutaneous squamous cell carcinoma (CSCC). A) Podoplanin expression in lymphatic vessels: (a1) Hematoxilin and eosin (H\&E) staining of the CSCC zone with lymphatic vessels (blue arrows) $(\times 400)$ and $(\mathrm{a} 2)$ increased magnification $(\times 400)$ showing more detail. B) Podoplanin expression in an infiltrative CSCC: (b1) H\&E of tumor cells $(\times 200)$; (b2) podoplanin expression in tumor cells showing cytoplasmic podoplanin staining $(\times 200)$ and $(\mathrm{b} 3)(\times 400)$. C) Podoplanin expression along the front of invasion in CSCC: $(\mathrm{c} 1)$ H\&E staining in an infiltrative CSCC $(\times 200)$. Podoplanin expression along the front of invasion of the tumor $(c 2)(\times 200)$ and (c3) $(\times 400)$.

Podoplanin predicts short disease-free relapse in CSCC

Later, we assessed whether podoplanin expression could serve as a biomarker of time to disease relapse in CSCC. The median follow-up period of all the patients was 70 months (range 36-90 months). The latency to any event of poor clinical evolution was shorter for those patients with tumors that expressed podoplanin at moderate/intense levels $(p=0.031)$ (Fig. 3A). Thus, the median length of time to develop adverse clinical events was 4.5 months [interquartile range (IQR) 6.5] for those patients with tumors that expressed podoplanin at moderate/intense levels, whereas it was 11 months (IQR 21) for those patients with tumors with absent/weak podoplanin expression (Table 1).

Also, moderate/intense podoplanin expression was associated with a shorter latency to develop lymph nodal metastasis $(\mathrm{p}=0.008)$ (Fig. 3B). Thus, the median length of time to NP was 5 months (IQR 4.88) for those tumors with moderate/intense podoplanin expression, and 13 months (IQR 18) for those with absent/weak podoplanin expression (Table 1).

We carried out a logistic regression model to evaluate the plausibility of podoplanin expression acting as an independent variable able to predict CSCC prognosis. We only found it to be associated with the lymph node metastasis model (Table 2). In addition to podoplanin expression, we introduced tumor traits related to CSCC prognosis and presumed to be predictable variables according to the literature. ${ }^{6,24,25}$ These included desmoplasia, growth pattern, perineural infiltration, tumor thickness, tumor size and degree of differentiation, some of which have been associated with poor prognosis by univariate analysis in our cohort (Tables S2 and S3). Only perineural infiltration and podoplanin expression were the variables associated with short disease-free time to relapse in our cohort. AJCC stage did not correlate with differences in the time to events and NP.

\section{Discussion}

In this study, we showed that moderate/intense podoplanin expression was associated with histopathological features of poor outcome in CSCC, with NP and a short latency to events of poor clinical evolution generally considered, and in particular, with nodal metastasis. ${ }^{26}$

We found podoplanin expression in lymphatic vessels, and in tumor cells where, in some cases, it exhibited cytoplasmic staining. The specific expression of podoplanin in lymphatic endothelial cells and tumor cells in CSCC is similar to 
Cañueto et al.

A
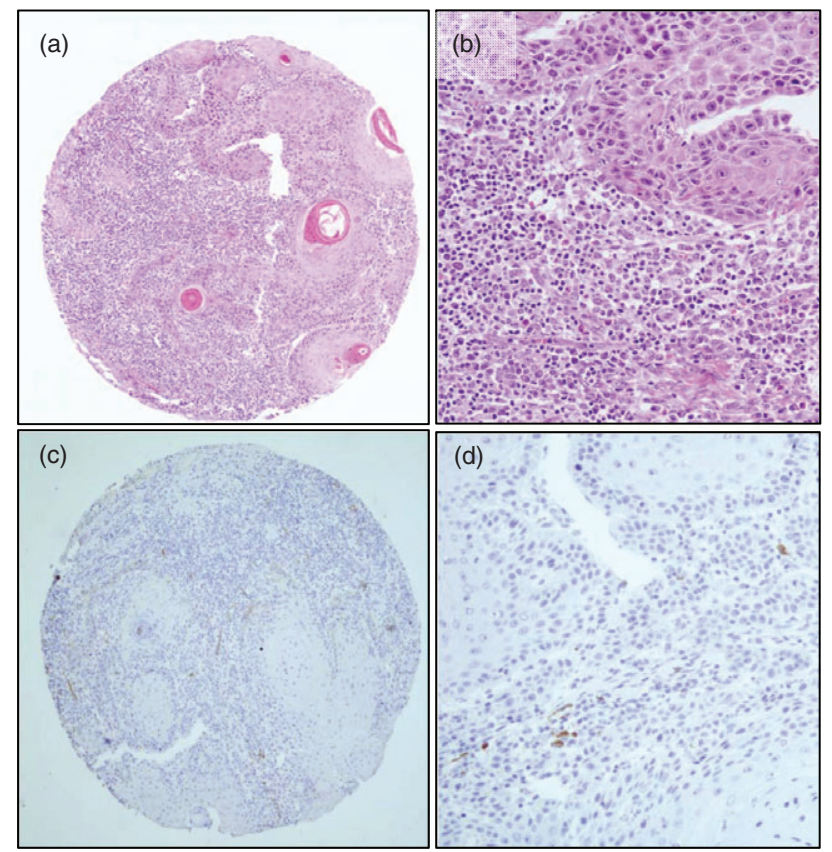

B
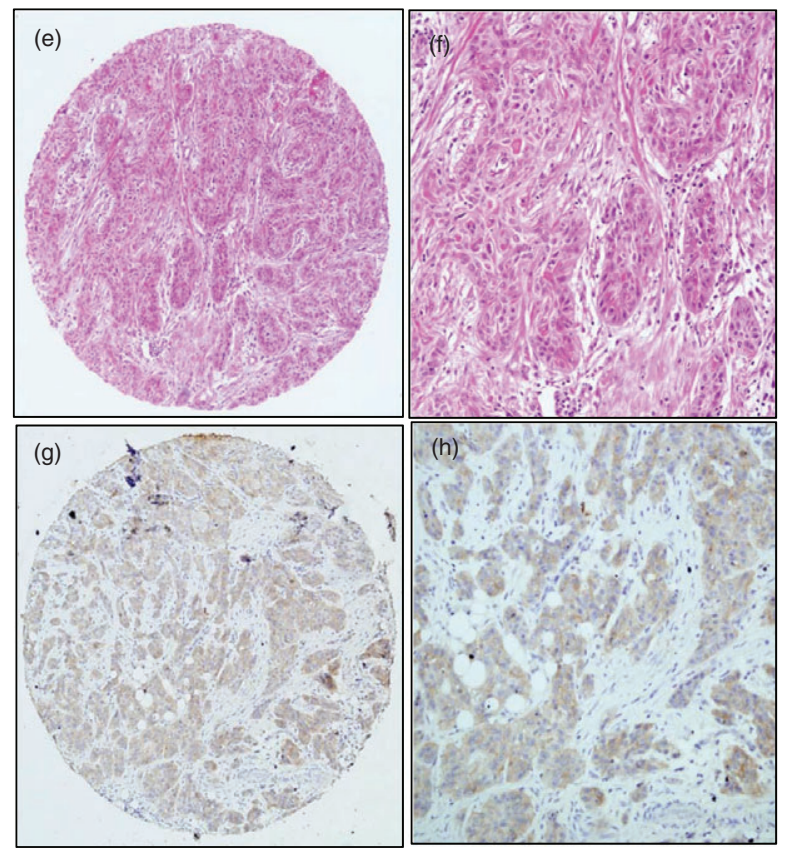

C

Podoplanin expression

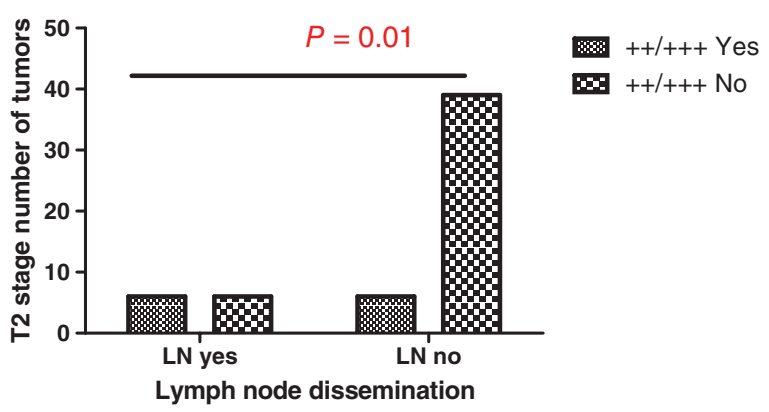

Fig. 2. Podoplanin expression and histopathological tumor traits in cutaneous squamous cell carcinoma (CSCC). A) Well-differentiated CSCCs with an expansive growth pattern of invasion: (a) H\&E ( $\times 100)$; (b) H\&E ( $\times 200)$; (c) absence of global podoplanin expression in the tumor $(\times 100)$; (d) note that lymphatic vessels are positively stained $(\times 200)$. B) Poorly differentiated CSCCs with infiltrative growth pattern of invasion; (e) H\&E ( $\times 100)$; (f) H\&E ( $\times 200)$; (g) intense podoplanin expression ( $\times 100)$ and $(\mathrm{h})(\times 200)$. Note the desmoplastic stroma in (f) and (h). C) Association of lymph node metastasis with podoplanin expression in tumors at stage 2 .

the expression pattern of podoplanin observed in other types of SCG such as the carcinoma of the oral cavity and the larynx. ${ }^{27-30}$ This finding is in agreement with the role of podoplanin in lymphatic vessel generation and remodeling, ${ }^{9}$ but there were some differences in the frequency and the intensity of podoplanin expression, being lower in CSCC. ${ }^{21}$ Indeed, we did observe that the expression of podoplanin was absent in more than $50 \%$ of the tumors in this cohort. As podoplanin expression seems to be linked with lymphatic metastases and with lymphatic tumor spread, these differences in podoplanin expression frequency might reflect the lower nodal metastatic risk in SCC of cutaneous origin. ${ }^{21}$ In addition, we observed an association between podoplanin expression in the primary tumor and the presence of lymphovascular invasion, which suggests that podoplanin is associated with lymphatic tumor spread. Podoplanin also tended to be expressed along the front of invasion of tumors, especially in those cases that exhibited an infiltrative growth pattern, and its expression has been associated with an infiltrative growth pattern and desmoplasia in CSCC. ${ }^{20}$

Podoplanin has been previously associated with nodal metastasis ${ }^{20,21,31}$ and with short survival rates in CSCC. ${ }^{32}$ Thus, podoplanin 
D2-40 in cutaneous squamous cell carcinoma prognosis

Table 1. Associations between podoplanin expression and different clinical and histopathological CSCC variables

\begin{tabular}{|c|c|c|c|c|}
\hline \multirow[b]{2}{*}{ CSCC features } & & \multicolumn{2}{|c|}{ Podoplanin expression } & \multirow[b]{2}{*}{ p Value } \\
\hline & & Absent/weak & Moderate/intense & \\
\hline \multirow[t]{2}{*}{ Desmoplasia } & Yes & 13 & 7 & $0.048^{* * *}$ \\
\hline & No & 62 & 10 & \\
\hline \multirow[t]{2}{*}{ Growth pattern } & Infiltrative & 37 & 14 & $0.013^{\star \star *}$ \\
\hline & Non infiltrative & 38 & 3 & \\
\hline \multirow[t]{2}{*}{ Ulceration } & Yes & 36 & 15 & $0.007^{* * *}$ \\
\hline & No & 26 & 1 & \\
\hline \multirow[t]{2}{*}{ Lymphovascular invasion } & Yes & 2 & 4 & $0.011^{*}$ \\
\hline & No & 72 & 13 & \\
\hline \multirow[t]{2}{*}{ Degree of differentiation } & Good-moderate & 63 & 12 & $0.057^{*}$ \\
\hline & Poor & 12 & 7 & \\
\hline \multirow[t]{2}{*}{ Nodal progression } & Yes & 7 & 6 & $0.022^{\star}$ \\
\hline & No & 68 & 13 & \\
\hline \multirow[t]{2}{*}{ AJCC stage at diagnosis } & I & 27 & 5 & N.S. ${ }^{* *}$ \\
\hline & II & 48 & 14 & \\
\hline \multirow[t]{4}{*}{ AJCC stage at the end of follow up } & I & 26 & 5 & $0.032^{\star * *}$ \\
\hline & II & 41 & 8 & \\
\hline & III & 7 & 3 & \\
\hline & IV & 1 & 3 & \\
\hline \multirow[t]{2}{*}{ Progression AJCC stage } & Yes & 8 & 6 & $0.033^{* * *}$ \\
\hline & No & 67 & 13 & \\
\hline \multicolumn{2}{|c|}{ Time to events of poor prognosis (months) (Me/IQR) } & $11(21)$ & $4.5(6.5)$ & $0.031^{\star *}$ \\
\hline \multicolumn{2}{|c|}{ Time to nodal progression (months) (Me/IQR) } & $13(18)$ & $5(3.5)$ & $0.008^{* *}$ \\
\hline
\end{tabular}

CSCC, cutaneous squamous cell carcinoma; IQR, interquartile range; Me, median; N.S. not significant.

Significant $p$ values are in bold; Statistical trend is indicated by italics.

${ }^{\star}$ Fisher exact test; ${ }^{* *}$ Mann-Whitney $U$ test; ${ }^{* * *}$ Chi-square test was used in those cases with no specification.

seems to be a marker associated with NP in CSCC. ${ }^{20,21}$ Here, we also found an association between nodal metastasis and moderate/intense podoplanin expression in CSCC. Although podoplanin expression has been associated with relapse in CSCC, we did not find an association between this marker and LR in our series. Other authors have found correlation between low podoplanin expression and lymphatic spread as well as with nodal metastases in uterine cervical cancer $^{33}$ and in lung cancer, ${ }^{34}$ and it seems podoplanin may play a different role depending on the cancer type.

The association of podoplanin expression with latency to events of bad clinical evolution has been poorly evaluated in CSCC. In our study, moderate/intense podoplanin expression was associated with a short latency to global events and with a short latency to NP. Indeed, podoplanin expression was the unique variable associated with short disease-free time of to relapse. AJCC stage at diagnosis was inadequate to predict latency to events of bad clinical evolution. Also podoplanin expression, which did not correlate with AJCC stage at diagnosis, did correlate with AJCC stage at the end of follow up and with the progression of AJCG stage. Thus, podoplanin expression could be a useful marker to identity tumors with a high risk of progression and could give additional prognostic information for CSCC AJCC stage at diagnosis, in particular for these tumors at stage 2 (Fig. 2C).

In conclusion, this paper confirms podoplanin as a marker of poor prognosis in CSCC. We show that podoplanin expression predicts a higher risk for the development of nodal metastasis in

Table 2. Identification of podoplanin expression and perineural infiltration as independent variables to predict lymph node metastasis by logistic regression

\begin{tabular}{lcccc}
\hline Logistic regression model & Independent factors & OR & $\mathrm{Cl}$ & $\mathrm{p} \mathrm{Value}$ \\
\hline Lymph node metastasis & PNI & 5.75 & $1.540-21.467$ & 0.009 \\
& Podoplanin $(++/+++)$ & 5.165 & $1.326-20.126$ & 0.018 \\
\hline
\end{tabular}

$(++/+++)$, moderate/intense; $\mathrm{Cl}$, confidence interval; OR, odd ratio; PNI, perineural infiltration. 

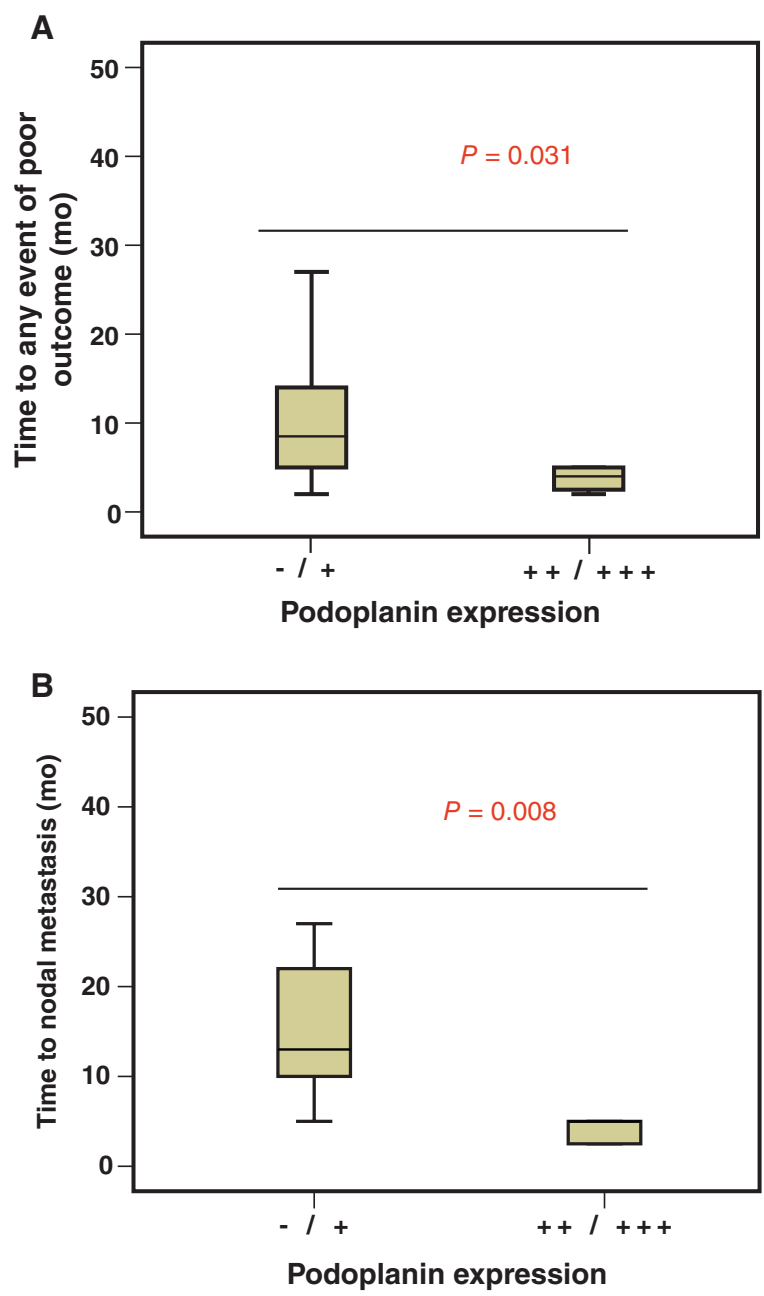

Fig. 3. Differences in disease-free time to relapse associated with podoplanin expression. A) podoplanin expression is associated with short disease-free time of relapse in cutaneous squamous cell carcinoma (CSCC). B) podoplanin expression is associated with a short latency to lymph nodal progression in CSCC.

CSCC, as others have also described,,$^{20,27}$ and is also a marker of an early appearance of a clinical event. This article provides evidence for the prognostic implications of podoplanin expression, and suggests that CSCCs with moderate/intense podoplanin expression should be closely followed irrespective of other associated risk factors. Moderate/intense expression of podoplanin in the primary tumor, especially in those cases with other pathological traits of aggressiveness, could justify carrying out a sentinel lymph node biopsy for better staging of CSCC. While evidence is growing concerning the prognostic implications of podoplanin expression in CSCC, further studies with larger case series are necessary to confirm these findings.

\section{Acknowledgements}

JPL laboratory is partially funded by FEDER and MICINN (PLE2009-119, SAF2014-56989-R), Instituto de Salud Carlos III (PI07/0057, PI10/00328, PIE14/00066), Junta de Castilla y León (CSI034U13, BIO/SA31/15, CSI001416), IBSAL (IBY15/00003), the Fundación 'Eugenio Rodríguez Pascual', the Fundación 'Inbiomed' (Instituto Oncológico Obra Social de la Caja Guipozcoa-San Sebastian, Kutxa) and the Fundación 'Sandra Ibarra de Solidaridad frente al Cáncer', Q3718001E (2009-2010) and GRS 612/A/11 (2011-2012). JC is partially funded by the Gerencia Regional de Salud (Junta de Castilla y León): GRS 1342/A/16.

\section{Supporting Information}

Additional Supporting Information may be found in the online version of this article:

Table S1. Clinicoepidemiological and histopathological features of cutaneous squamous cell carcinoma (CSCC) evaluated in our cohort of study.

Table S2. Associations between histopathological tumor traits of cutaneous squamous cell carcinoma (CSCC) with events of poor clinical outcome.

Table S3. Associations of cutaneous squamous cell carcinoma (CSCC) prognosis and podoplanin expression with (A) presence of immunosuppression; (B) location of CSCC in high-risk zones and $(\mathrm{C})$ tumor thickness and size.

\section{References}

1. Motley R, Kersey P, Lawrence C. Multiprofessional guidelines for the management of the patient with primary cutaneous squamous cell carcinoma. Br J Dermatol 2002; 146: 18.

2. Preston DS, Stern RS. Nonmelanoma cancers of the skin. N Engl J Med 1992; 327: 1649.

3. Karia PS, Han J, Schmults CD. Cutaneous squamous cell carcinoma: estimated incidence of disease, nodal metastasis, and deaths from disease in the United States, 2012. J Am Acad Dermatol 2013; 68: 957.
4. Miller DL, Weinstock MA. Nonmelanoma skin cancer in the United States: incidence. J Am Acad Dermatol 1994; 30 (5 Pt 1): 774 .

5. Housman TS, Feldman SR, Williford PM, et al. Skin cancer is among the most costly of all cancers to treat for the Medicare population. J Am Acad Dermatol 2003; 48: 425.

6. Brantsch KD, Meisner C, Schonfisch B, et al. Analysis of risk factors determining prognosis of cutaneous squamous-cell carcinoma: a prospective study. Lancet Oncol 2008; 9: 713.
7. Carter JB, Johnson MM, Chua TL, Karia PS, Schmults CD. Outcomes of primary cutaneous squamous cell carcinoma with perineural invasion: an 11-year cohort study. JAMA Dermatol 2013; 149: 35.

8. Karia PS, Jambusaria-Pahlajani A, Harrington DP, Murphy GF, Qureshi AA, Schmults CD. Evaluation of American Joint Committee on Cancer, International Union Against Cancer, and Brigham and Women's Hospital tumor staging for cutaneous squamous cell carcinoma. J Clin Oncol 2013; 32: 327. 


\section{D2-40 in cutaneous squamous cell carcinoma prognosis}

9. Schacht V, Dadras SS, Johnson LA, Jackson DG, Hong YK, Detmar M. Up-regulation of the lymphatic marker podoplanin, a mucin-type transmembrane glycoprotein, in human squamous cell carcinomas and germ cell tumors. Am J Pathol 2005; 166: 913.

10. Wicki A, Christofori G. The potential role of podoplanin in tumour invasion. $\mathrm{Br} \mathrm{J}$ Cancer 2007; 96: 1.

11. Gandarillas A, Scholl FG, Benito N, Gamallo C, Quintanilla M. Induction of PA2.26, a cell-surface antigen expressed by active fibroblasts, in mouse epidermal keratinocytes during carcinogenesis. Mol Carcinog 1997; 20: 10.

12. Fernandez-Munoz B, Yurrita MM, Martin-Villar E, et al. The transmembrane domain of podoplanin is required for its association with lipid rafts and the induction of epithelial-mesenchymal transition. Int J Biochem Cell Biol 2011; 43: 886 .

13. Wicki A, Lehembre F, Wick N, Hantusch B, Kerjaschki D, Christofori G. Tumor invasion in the absence of epithelial-mesenchymal transition: podoplanin-mediated remodeling of the actin cytoskeleton. Cancer Cell 2006; 9: 261.

14. Martin-Villar E, Scholl FG, Gamallo C, et al. Characterization of human PA2.26 antigen (T1alpha-2, podoplanin), a small membrane mucin induced in oral squamous cell carcinomas. Int J Cancer 2005; 113: 899.

15. Funayama A, Cheng J, Maruyama S, et al. Enhanced expression of podoplanin in oral carcinomas in situ and squamous cell carcinomas. Pathobiology 2011; 78: 171.

16. Inoue $\mathrm{H}$, Miyazaki $\mathrm{Y}$, Kikuchi $\mathrm{K}$, et al. Podoplanin expression during dysplasia-carcinoma sequence in the oral cavity. Tumour Biol 2012; 33: 183.

17. Liang $\mathrm{H}, \mathrm{Wu} \mathrm{H}$, Giorgadze $\mathrm{TA}$, et al. Podoplanin is a highly sensitive and specific marker to distinguish primary skin adnexal carcinomas from adenocarcinomas metastatic to skin. Am J Surg Pathol 2007; 31: 304.

18. Tong L, Yuan S, Feng F, Zhang H. Role of podoplanin expression in esophageal squamous cell carcinoma: a retrospective study. Dis Esophagus 2012; 25: 72.

19. Guimaraes GC, Lopes A, Campos RS, et al. Front pattern of invasion in squamous cell carcinoma of the penis: new prognostic factor for predicting risk of lymph node metastases. Urology 2006; 68: 148

20. Toll A, Gimeno-Beltran J, Ferrandiz-Pulido C, et al. D2-40 immunohistochemical overexpression in cutaneous squamous cell carcinomas: a marker of metastatic risk. J Am Acad Dermatol 2012; 67: 1310.

21. Kreppel M, Krakowezki A, Kreppel B, et al. Podoplanin expression in cutaneous head and neck squamous cell carcinoma - prognostic value and clinicopathologic implications. J Surg Oncol 2013; 107: 376 .

22. Johnson TM, Rowe DE, Nelson BR, Swanson NA. Squamous cell carcinoma of the skin (excluding lip and oral mucosa). J Am Acad Dermatol 1992; 26(3 Pt 2): 467.

23. Metchnikoff C, Mully T, Singer JP, Golden JA, Arron ST. The 7th edition AJCC staging system for cutaneous squamous cell carcinoma accurately predicts risk of recurrence for heart and lung transplant recipients. J Am Acad Dermatol 2012; 67: 829.

24. Cherpelis BS, Marcusen C, Lang PG. Prognostic factors for metastasis in squamous cell carcinoma of the skin. Dermatol Surg 2002; 28: 268

25. Frierson HF Jr, Cooper PH. Prognostic factors in squamous cell carcinoma of the lower lip. Hum Pathol 1986; 17: 346.

26. Veness MJ, Palme CE, Morgan GJ. High-risk cutaneous squamous cell carcinoma of the head and neck: results from 266 treated patients with metastatic lymph node disease. Cancer 2006; 106: 2389.
27. Kreppel M, Drebber U, Wedemeyer I, et al. Podoplanin expression predicts prognosis in patients with oral squamous cell carcinoma treated with neoadjuvant radiochemotherapy. Oral Oncol 2011; 47: 873.

28. Kreppel M, Scheer M, Drebber U, Ritter L, Zoller JE. Impact of podoplanin expression in oral squamous cell carcinoma: clinical and histopathologic correlations. Virchows Arch 2010; 456: 473 .

29. Vormittag L, Thurnher D, Geleff S, et al. Co-expression of Bmi-1 and podoplanin predicts overall survival in patients with squamous cell carcinoma of the head and neck treated with radio(chemo) therapy. Int J Radiat Oncol Biol Phys 2009; 73: 913.

30. Yuan P, Temam S, El-Naggar A, et al. Overexpression of podoplanin in oral cancer and its association with poor clinical outcome. Cancer 2006; 107: 563.

31. Toll A, Masferrer E, Hernandez-Ruiz ME, et al. Epithelial to mesenchymal transition markers are associated with an increased metastatic risk in primary cutaneous squamous cell carcinomas but are attenuated in lymph node metastases. J Dermatol Sci 2013; 72: 93.

32. Vinicius de LV, Scapulatempo C, Perpetuo NM, et al. Prognostic and risk factors in patients with locally advanced cutaneous squamous cell carcinoma of the trunk and extremities. J Skin Cancer 2011; 2011: 420796.

33. Dumoff KL, Chu CS, Harris EE, et al. Low podoplanin expression in pretreatment biopsy material predicts poor prognosis in advanced-stage squamous cell carcinoma of the uterine cervix treated by primary radiation. Mod Pathol 2006; 19: 708.

34. Shimada Y, Ishii G, Nagai K, et al. Expression of podoplanin, CD44, and p63 in squamous cell carcinoma of the lung. Cancer Sci 2009; 100: 2054. 